\section{Recruitment and participation in pre-school and school-based fluoride varnish pilots - the South Central experience}

\author{
S. Buckingham ${ }^{* 1}$ and J. H. John²
}

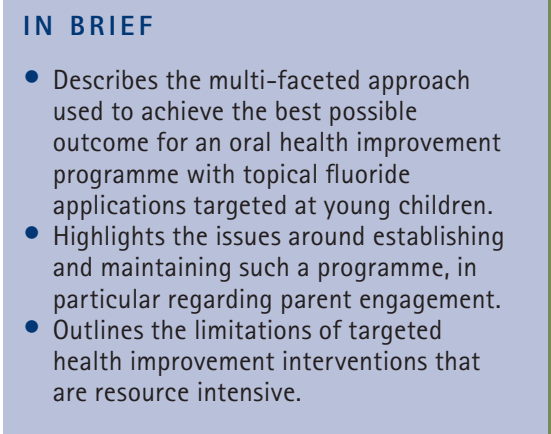

Objective To assess recruitment and participation within seven school/pre-school-based programmes for fluoride varnish applications. Method Year-long pilots were undertaken in six primary schools (ages four to seven years) and one pre-school (ages three to four years). Three applications of fluoride varnish were carried out coinciding with the three school terms. Results In spite of intensive recruitment efforts, only 78\% of the total 589 children were enrolled in the pilot; 15\% had to be excluded because no response could be obtained from their parents. At the end of 12 months, 79\% of enrolled children (62\% of total) had received all three applications. Children did not receive applications because they were absent on the days when the dental team were carrying out the screening or fluoride varnish applications, were ill, or because the child refused. The highest refusal rate was in the pre-school. Conclusions Younger children need to be targeted if improvement in the oral health of five-year-olds is to be achieved, the only dental measure in the Public Health Outcomes Framework, but they were less likely to cooperate. More research is needed on approaches to maximise participation in community programmes such as this, if they are to achieve significant population-level improvements in child dental health.

\section{INTRODUCTION}

Over the past 20 years there has been an overall decline in the incidence of dental caries in children. However, inequalities remain, with those from the most deprived backgrounds experiencing the highest levels of disease. ${ }^{1,2}$

Fluoride is known to reduce the incidence of dental caries and has been shown to have a substantial caries-inhibiting effect. ${ }^{3,4}$ Fluoridated toothpaste, which has been widely available to the population since the 1970s, has played a major role in improvements to dental health at population level. Evidence shows that 'application of fluoride varnishes two to four times a year, either in the permanent or deciduous dentition, is associated with substantial reduction in caries increment. ${ }^{3}$ The Department of

Specialist in Special Care Dentistry, Oxfordshire Salaried Primary Care Dental Service, Oxford Health NHS Foundation Trust, Astral House, Granville Way, Bicester, Oxfordshire, OX26 4JT; ${ }^{2}$ Consultant in Dental Public Health, Southampton, Hampshire, Isle of Wight and Portsmouth PCT Cluster, Oakley Road, Southampton, S016 4GX

${ }^{*}$ Correspondence to: Sarah Buckingham

Email: sarah.buckingham@oxfordhealth.nhs.uk

\section{Online article number E8}

Refereed Paper - accepted 28 March 2013

DOI: $10.1038 /$ sj.bdj.2013.834

British Dental Journal 2013; 215: E8
Health document Delivering better oral health - an evidence-based toolkit ${ }^{5}$ (DBOH) provides dental practitioners with continually reviewed evidence-based recommendations on how to improve many aspects of oral health, including increasing the availability of fluoride to the population. DBOH recommends two to four applications a year from the age of 3 years to 16 years depending on the patients' assessed risk of caries. One-off or ad-hoc applications are ineffective in achieving long-term benefit. It is known that children who are at highest risk are least likely to attend regularly and so do not access these benefits. Over the last few years community oral health improvement programmes involving the use of topical fluoride varnishes have been set up across the United Kingdom to try and reach all children. National programmes include the 'Childsmile' programme in Scotland ${ }^{6}$ and the 'Designed to Smile' programme in Wales. ${ }^{7}$ The South Central region oral health improvement pilots were set up with the aim of testing the feasibility and acceptability of an oral health promotion programme with topical fluoride varnish applications in a local pre-school and schools based on DBOH Department of Health (DH) guidance. ${ }^{5}$ The objectives of the pilots were to assess recruitment and retention rates and identify the main factors affecting the success of these programmes. This paper reports on the experience of establishing and recruiting to the year-long pilots.

\section{METHODS}

School pilots were set up for a year initially, across three areas in the South Central region, Oxfordshire (two schools), Portsmouth (three schools) and Southampton (one school and one preschool). The children were aged between four and seven years in the schools and between three and four years in the preschool. A pre-school/school setting was chosen to facilitate access and follow-up of participants. Children were recruited from reception year in the schools (four to five years of age) and the entry year in the pre-school (three years of age). All the schools involved were very supportive of the pilots. The schools were located in areas of deprivation and drew their children from the surrounding neighbourhoods. Data from the NHS dental epidemiological surveys, ${ }^{8}$ local public health deprivation data and local intelligence identified the children in these schools as presenting with 'high dental needs'. A 
postcode analysis of individual children to determine any association between deprivation and participation was therefore not included as almost all the children were from deprived backgrounds.

In accordance with Department of Health guidance, the consultant in dental public health (CDPH) from the Hampshire and Isle of Wight region oversaw all the pilots. During the year, the CDPH facilitated a continuous flow of information between the pilots about issues and best practice to maximise a successful outcome for all sites. Each area had a team with an extended-duty dental nurse managing the intervention at the school, including providing fluoride varnish applications, ${ }^{9}$ supported by an oral health promotion team that included a dentist for screening and a dentist or dental therapist providing clinical support. A holistic multi-faceted approach that included dietary education, daily supervised toothbrushing sessions, oral health training for staff and parental engagement was used.

All children who were enrolled in the pilots were screened initially by a dentist to identify any dental decay that needed follow-up care and to prescribe the fluoride varnish. Three applications of fluoride varnish were planned, a pragmatic decision that was within the guidance and conformed to the three school terms to facilitate planning with the school. The fluoride varnish was applied by an extended-duty dental nurse trained in fluoride varnish application. The first application was carried out at the screening appointment. Medical histories were updated using reminder letters and telephone calls before every topical fluoride varnish application.

As anticipated, the biggest challenge was recruitment to the pilots and significant efforts were made using a variety of methods to maximise participation. Examples included poster displays to publicise the programme, a 'signing day' for returning signed consent forms, text reminders and intercepting parents before and after school.

School and dental staff worked together to encourage children to accept the applications using a variety of methods, including role play. Children who were recruited to the pilots were screened before the first application of fluoride varnish. Repeat screening sessions were organised for each application to maximise participation. Written information about the application and any treatment needed was sent to parents and to the child's dentist, if they had one. Appointments within the salaried dental services were offered to children who required dental treatment but were not currently receiving regular dental care.

\section{RESULTS}

The total number of children in the seven sites was 589. A completed consent form was received from 503 (85.4\%). The response rate from sending the consent forms home in the children's book bags was very poor, with only 20-30\% at each site returning completed consent forms. Even with the intensive efforts, including repeated follow-ups, the biggest loss to recruitment was non-response to recruitment letters. A total of $86(14.6 \%)$ children were lost in this way. Of the 503 who returned the forms, only 16 (3.2\%) declined consent. Of the consented children, 22 were not enrolled as the fluoride varnish was contraindicated for 21 due to their medical history and one child was already receiving applications at a dental practice. Seven children left the school between the consent process and the start of the programme. A total of 458 children $(77.8 \%$ of the total 589 children) were enrolled in the pilot year, of whom 363 (61.6\% of the total 589 children) received the three applications as planned. Figure 1 provides a flow chart of children's participation, while Figure 2 demonstrates the decline in participating children at each stage of the programme.

Missed sessions were generally due to absences, illness or child refusals on the day, either for the screening or the fluoride varnish application. Child refusals were highest in the pre-school group.

\section{DISCUSSION}

These South Central pilots were set up in line with the evidence that states that increasing the availability of fluoride, through fluoride varnish applications, contributes to reduction in dental caries risk. ${ }^{3-5}$ However, children who do not attend regularly for dental care miss out, and delivering it in a school setting seems a good option to reach all children. The programme included dietary advice for parents and teachers and supervised toothbrushing sessions to achieve the best outcome.
Overall, the programme was very resource-intensive. Staff in the pre-school and primary schools were supportive throughout, but continued engagement was needed to maintain their motivation. It was difficult for staff to find time in their busy schedules for discussions and training.

As anticipated, recruitment was the most challenging aspect of the pilot. Engaging with parents was difficult. There was little interest in parents' evenings, which meant that the dental teams had to be on-site for one-to-one discussions with parents before and after school. This was not always successful as parents were often unable to stay for long and many children were not accompanied by their parents. Some parents had difficulties understanding the consent form even with explanations due to language and literacy barriers. There were some parents who were unwilling to engage and some who could not be reached at all, even after multiple attempts using a variety of methods. The dental team had to update medical histories before each application, which presented the same issues with reaching parents on each occasion.

Even with this dedicated and intensive approach, the teams were able to enrol less than $80 \%$ of the children and only about two-thirds of the original cohort received all three planned applications. There is concern that those children who were not included may be those with dental decay or at highest risk of dental decay and therefore would have benefited the most, even in this 'high-needs' population.

The pilots included three-year-olds in a pre-school to assess the feasibility of providing fluoride varnish applications earlier in life so as to influence dental outcomes for five-year-olds. The dental decay rate for five-year-olds is the only oral health measure included in the new Public Health Outcomes framework. ${ }^{10,11}$ Targeting younger children may also have a greater impact on reducing the risk of dental decay and encourage positive attitudes from an earlier age. However, the greatest number of refusals from children for screening and fluoride varnish applications was in the pre-school. The younger children required greater engagement and the application process was more time-consuming. Repeat attempts were made with some of the children who refused. This was not always deemed possible to avoid upsetting 
children, nor was it always successful. Two of the younger children refused the screening and had to be excluded from the programme, which meant that they did not receive any fluoride varnish applications.

In Scotland the Childsmile programme includes both fluoride varnish applications and school toothbrushing programmes. ${ }^{6}$ Monitoring of the programme through its first three years from 2006 to 2009 has shown considerable variation in consent rates for fluoride varnish applications across the schools. The overall coverage was only 47\% of the P1 and P2 school rolls (4- to 7-year-olds). Further analysis is underway to assess if social deprivation is related to participation in this programme. There have been significant improvements to child oral health in Scotland ${ }^{1}$ that may be primarily due to the establishment of supervised toothbrushing programmes nationally, but even these have seen a falloff after the first year at primary school. ${ }^{12}$ By the end of the 2008-2009 school year $95 \%$ of all Scottish nursery schools attended by 97,000 three to five-year-olds were participating in supervised toothbrushing, however, this participation rate decreased in P1 and P2. ${ }^{13}$ The Childsmile programme is currently being evaluated and it will be interesting to see if the trajectory of oral health improvement achieved is sustainable in the long-term. ${ }^{14,15}$

Designed to Smile is a similar programme in Wales delivering supervised toothbrushing programmes and fluoride varnish applications. ${ }^{7}$ Dental treatments are delivered at nursery and school sites using a mobile dental vehicle. It has only recently been introduced and is yet to be evaluated.

Supervised toothbrushing schemes have been established in schools in England for some years since the DH 'Brushing for Life'16 initiative some years ago. The national initiative has now ended, but supervised toothbrushing programmes continue throughout the country. There is no national oral health improvement programme involving fluoride varnish applications in England. It is not known if these types of programmes will achieve long-term population-wide improvements in oral health, over and above the supervised toothbrushing programmes. A recent trial in East Lancashire failed to demonstrate an effect for fluoride varnish applications when used as a public health intervention to prevent caries in

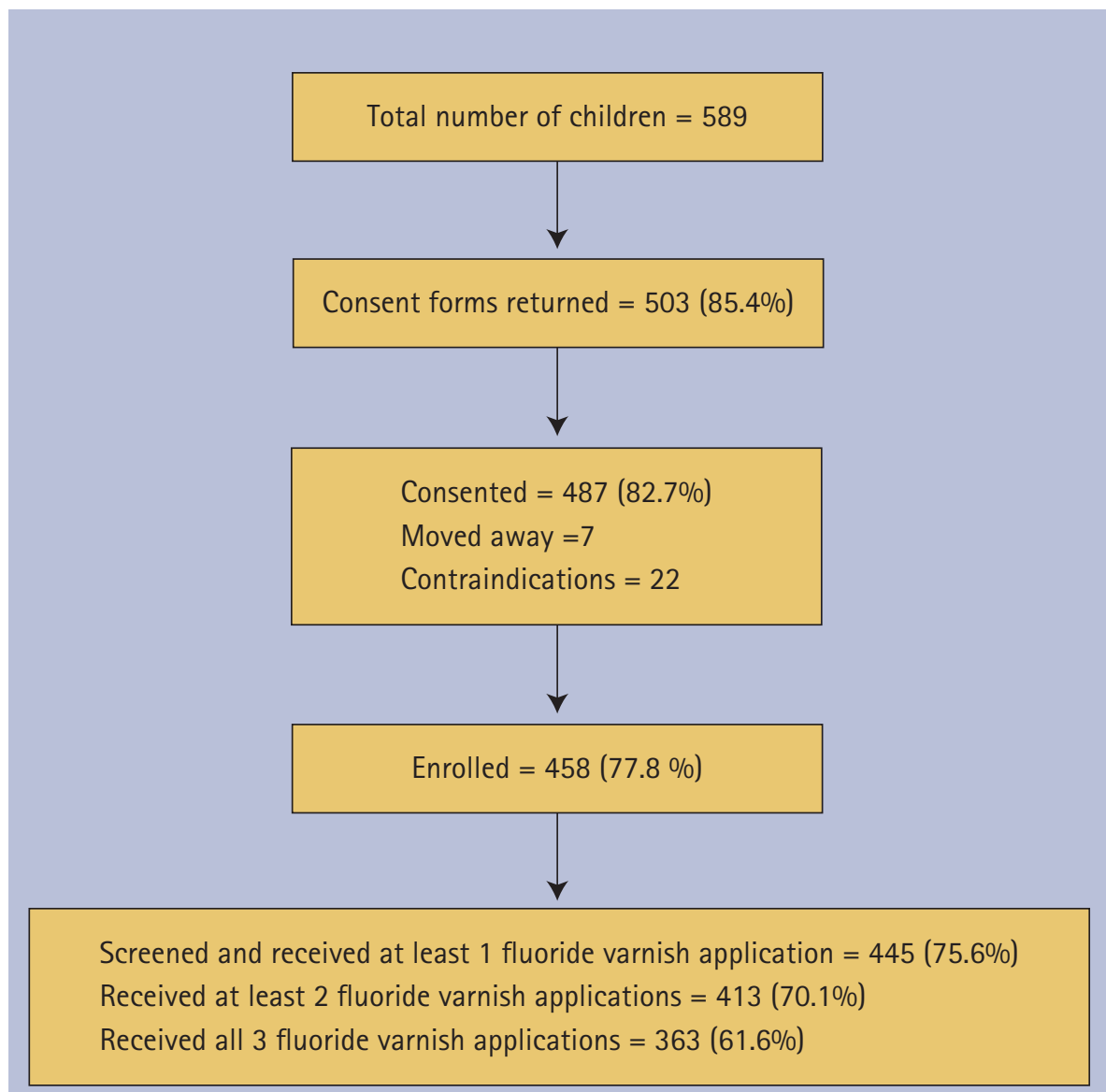

Fig. 1 Flow chart of children's participation

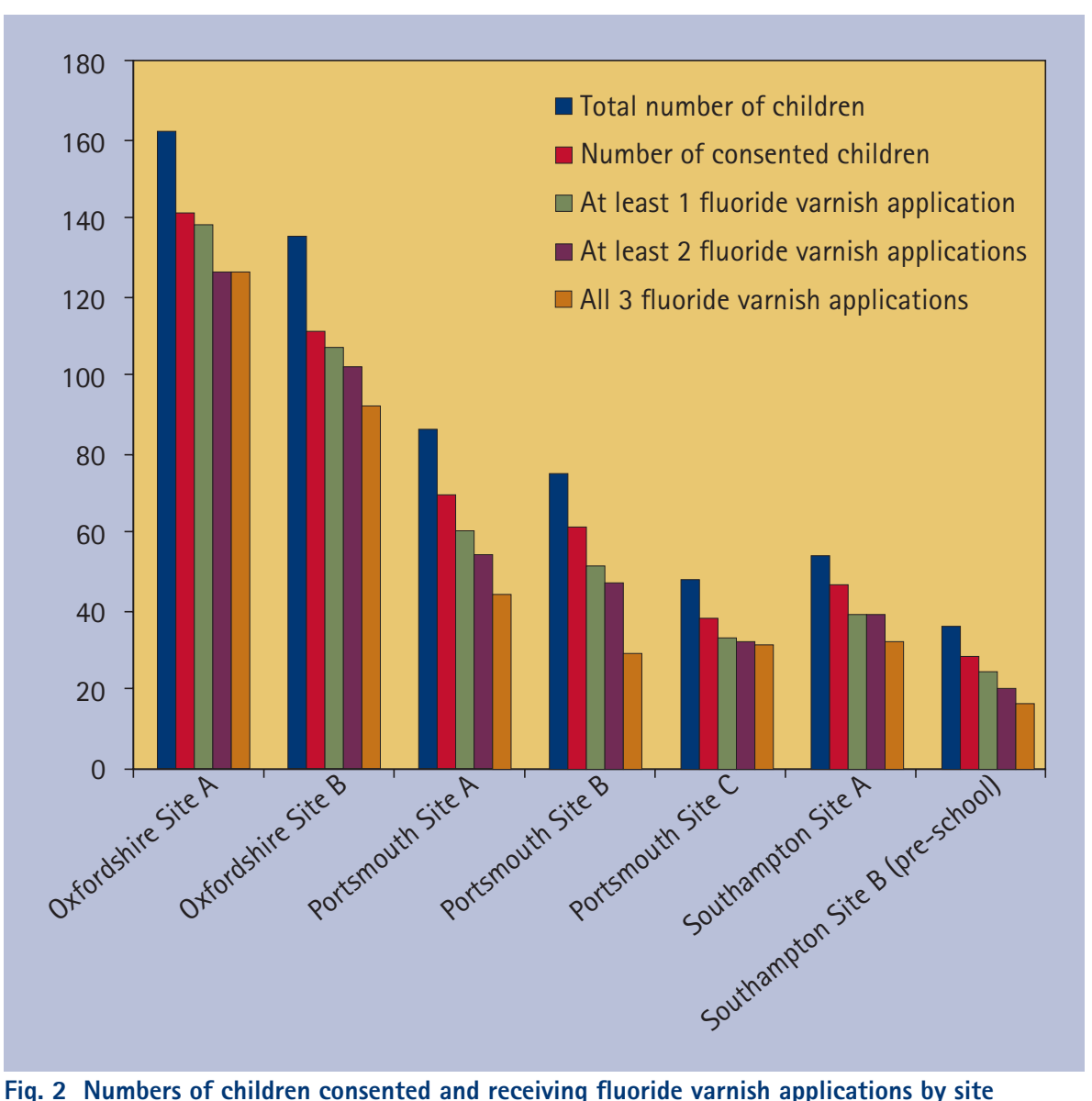

Fig. 2 Numbers of children consented and receiving fluoride varnish applications by site 
first permanent molar teeth. ${ }^{17}$ The authors reported a 50\% consent rate for their cohort and postulated that the low consent rates may have affected the effectiveness of the intervention. The authors' state: 'This was a pragmatic trial, and although providing consent for a trial is different from providing consent for a public health program, it is obvious that low consent rates reduce the effectiveness of public health interventions. ${ }^{18}$ These results concur with a study done in Manchester, ${ }^{19}$ which also failed to demonstrate any reduction in dental caries with twice-yearly applications of fluoride varnish in 6- to 8-year-olds. The Manchester study reported a consent rate of $51 \%$. Their trial cohort, with a mean age of 6.9 years, had a caries level that was lower than would be expected for the population. The decayed, missing and filled teeth (dmft) for a younger cohort of five-yearolds in $1999 / 2000$ was $2.9^{20}$ and the $\mathrm{d}_{3} \mathrm{mft}$ (dentine lesions) was 2.48 . The $\mathrm{d}_{3} \mathrm{mft}$ of the trial cohort was 2.39, which is lower rather than higher as would be expected for older children. The authors concluded that the "relatively low level of caries reduced the effectiveness of the intervention, since the children who participated were least likely to have benefited from the programme, whereas those who might have benefited did not respond when consent was sought:. ${ }^{19}$

In the South Central pilots there was a noticeable drop in numbers between consented children and those receiving the fluoride varnish applications. There were also a varying number of children at each of the three planned applications. This variable number of children at application visits resulted in approximately $80 \%$ of the enrolled children, two thirds $(60 \%)$ of the total number of children in the schools, having all three planned fluoride applications. The main reasons for this drop were absences and refusals. Repeat visits were organised to include as many children as possible but there is a limit to the number of times a dentist or dental nurse can return to reach absent children, particularly with the high rates of absenteeism in some of the schools. It was more time-consuming to carry out the applications in the pre-school and the refusal rate was higher. These younger children need to be targeted if the dental decay rate of five-year-olds is to be improved, but this type of intervention in younger children requires an increased investment of resources.

Supervised toothbrushing programmes in England, Scotland and Wales have been successfully implemented within schools and nurseries. ${ }^{16}$ They encourage the use of fluoride toothpaste and may prove to be more successful in increasing exposure to fluoride than fluoride varnish applications. However, even supervised toothbrushing programmes require cooperation from busy school and nursery staff and consent from all parents to achieve maximum coverage.

The best chance of improving dental health in children requires the use of a variety of interventions including dietary change, daily toothbrushing with the use of fluoride toothpaste and encouraging regular dental visits for the application of topical fluoride varnish and fissure sealants. These programmes need to be maintained through many years of school life to achieve longterm benefits. Impact and sustainability are important considerations for any health improvement programme for children.

The feasibility of implementing targeted oral health improvement programmes more widely needs to be carefully evaluated with respect to investment and outcomes. Targeted programmes are resource intensive and need to reach all children to be able to achieve significant improvements in children's dental health at populationlevel to justify the high level of investment. Population-wide improvements will not be achieved if children with the highest needs do not participate in these initiatives. This could, instead, lead to an undesirable widening in the dental health inequalities. ${ }^{21}$

\section{CONCLUSION}

Fluoride varnish applications have been shown to be effective in reducing risk of dental decay in research settings, but studies carried out in community settings in England have not achieved the same benefits. This may be due to issues with reaching all children, including those at highest risk of dental decay. Consideration needs to be given to the fact that implementing and maintaining oral health improvement programmes with topical fluoride varnish applications in community settings is resource intensive. More research is needed to determine how best to achieve participation of all children, if these programmes are to achieve measurable population-level improvements.
The authors are grateful for support from $\mathrm{Dr}$ Rosalind Mitchell (Clinical Director, Oxfordshire Salaried Primary Care Dental Service), Dr Denise Mattin (Clinical Director, Solent NHS Trust Dental Service) and Dr Andrew Mortimore (Director of Public Health, Southampton), without which this project would not have been possible.

1. Office of National Statistics. 2003 Children's Dental Health Survey. London: OFNS, 2004

2. Sabbah W, Tsakos G, Chandola D, Sheiham A, Watt $R$ G. Social gradients in oral and general health. J Dent Res 2007: 86: 992-996.

3. Marinho V C, Higgins J P, Logan S, Sheiham A. Fluoride varnishes for preventing dental caries in children and adolescents. Cochrane Database Syst Rev2002: CD002279.

4. Marinho V C, Higgins J P, Shelham A, Logan S. Combinations of topical fluoride (toothpastes, mouthrinses, gels, varnishes) versus single topical fluoride for preventing dental caries in children and adolescents. Cochrane Databse Syst Rev 2004; (1): CD002781.

5. Department of Health. Delivering better oral health an evidence-based toolkit for prevention. 2 nd ed. London: DH, 2009.

6. Childsmile. Improving the oral health of children in Scotland. Online information available at http:// www.child-smile.org.uk/ (accessed May 2013).

7. Designed to Smile. The Designed to Smile programme. Online information available at http:// www.designedtosmile.co.uk/home.html (accessed May 2013).

8. NHS Dental Epidemiology Programme for England. Oral health survey of five year old children 2007/2008. NHS Dental Epidemiology Programme for England, 2009. Online report available at http://www.nwph.net/ dentalhealth/reports/NHS_DEP_for_England_OH Survey_5yr_2007-08_Report.pdf (accessed May 2013).

9. NHS Primary Care Commissioning. The use of fluoride varnish by dental nurses to control caries. London: Department of Health, 2009. http://www.pcc-cic.org. uk/sites/default/files/articles/attachments/the use of fluoride varnish.pdf (accessed May 2013).

10. Department of Health. Healthy lives, healthy people: our strategy for public health in England. London: DH, 2010.

11. Department of Health. Healthy lives, healthy people: update and the way forward. London: DH, 2011.

12. McMahon A D, Blair Y, McCall D, MacPherson L M. Reductions in dental decay in 3-year old children in Greater Glasgow and Clyde: repeated population inspection studies over four years. BMC Oral Health 2011; 11: 29.

13. Scottish Government. Preschool and childcare census 2009. Edinburgh: Scottish Government, 2009.

14. MacPherson L M, Ball G.E, Brewster L et al. Childsmile: the national child oral health improvement programme in Scotland. Part 1: establishment and development. Br Dent J 2010; 209: 73-78.

15. Turner S, Brewster L, Kidd J et al. Childsmile: the national child oral health improvement programme in Scotland. Part 2: monitoring and delivery. Br Dent J 2010; 209: 79-83.

16. Blinkhorn A S. An appraisal of Brushing for Life. London: Department of health, 2008.

17. Milsom K M, Blinkhorn A S, Walsh T et al. A clusterrandomised controlled trial: Fluoride varnish in school children. J Dent Res 2011; 90: 1306-1311.

18. Spleith $\mathrm{CH}$, Steffen $H$, Welk $A$, Schwahn C. Responder and non-responder analysis for a caries prevention program. Caries Res 2005; 39: 269-272.

19. Hardman M C, Davies G M, Duxbury J T, Davies R M A cluster randomized controlled trial to evaluate the effectiveness of fluoride varnish as a public health measure to reduce caries in children. Caries Res 2007; 41: 371-376.

20. Pitts B, Evans D J, Nugent Z J. The dental caries experience of 5-year-old children in Great Britain: surveys coordinated by the British Society for the Study of Community Dentistry in 1999/2000. Community Dent Health 2001; 18: 49-55.

21. Schou L, Wight C. Does dental health education affect inequalities in dental health? Community Dent Health 1994; 11: 97-100. 